\title{
TRAPPED MODES IN TESLA CAVITIES*
}

\author{
$\underline{\text { F. Marhauser }}^{\ddagger}$, P. Hülsmann, H. Klein \\ Institut für Angewandte Physik der Johann Wolfgang Goethe-Universität Frankfurt \\ Robert Mayer-Straße 2-4, 60325 Frankfurt am Main, Germany
}

\begin{abstract}
In the TESLA linear collider scheme [1] the existence of trapped modes [2] within a TESLA cavity could be a severe problem to be overcome. Damping of trapped modes seems impossible due to their localization within the cavity, which prevents a coupling to the HOM dampers mounted to both ends of the beam pipes. As a consequence the energy of a trapped mode can achieve considerable values after several bunch passings, even in the case of a low shuntimpedance of such a mode. This effect gives additional heat load for the cooling system that has to be taken into account. In this paper we propose a method to prove the existence of these modes in a 9-cell copper TESLA cavity. RF-measurements with beam pipes and numerical simulations with MAFIA have been done. Longitudinal and transversal shuntimpedances of the trapped modes within a frequency range up to $10 \mathrm{GHz}$ were calculated.
\end{abstract}

\section{INTRODUCTION}

In the proposed TESLA linear collider scheme longitudinal and transversal emittance growth in the linac is required to be kept small. Furthermore the heating of the cavity walls due to wakefields in the low and high frequency regime has to be limited. One source of wall heating in the low frequency regime seems to be trapped modes.

Trapped modes are caused by variations of the cell geometry of the TESLA cavity. The usual TESLA cavity has almost equally shaped cells except the end cells. Due to the presence of the beam pipes these end cells differ slightly from the middle cells to ensure a flat field distribution for the accelerating mode. Thus trapped modes within the TESLA cavity and within the beam pipes, beyond and above the related cutoff frequency of the beam pipe, are expected. Two HOM dampers are foreseen for each single TESLA cavity, mounted to the beam pipes (length $=3 / 2 \lambda_{\text {fund }}$ ) on both ends nearby the end cells of the cavity. Consequently trapped modes within the beam pipes seem to be harmless because of their coupling to the HOM dampers.

\footnotetext{
*Work supported by the Graduiertenkolleg "Physik und Technik von Beschleunigern" der Deutschen Forschungsgemeinschaft DFG and by BMBF under contract 06OF841

Email: frankmar@sunkist.physik.uni-frankfurt.de
}

But trapped modes within the cavity have vanishing field amplitudes in the end cells of the cavity and therefore only low or even no coupling to the HOM dampers is expected. Usually trapped modes have only a small characteristic impedance (R/Q per cavity) of several ohms up to several ten ohms for the highest R/Q-values. From the viewpoint of particle dynamics those modes seem to be harmless, but from the viewpoint of stored energy a power of several ten up to several hundred watts is radiated into those modes and the time between two macropulses is too short to damp the stored modes energy significantly. This accumulation of wakefield energy could lead to a quench in the worst possible case.

To prove the existence of these trapped modes RFmeasurements were performed. Also numerical calculations have been done, with different boundary conditions, and have been compared with measurement results. Hence artifical trapped modes caused by the presence of the measurement antennas or other wall perturbations are out of question.

\section{MEASUREMENT AND SIMULATION}

\subsection{The RF-Test-Setup}

As mentioned above trapped HOMs within the cavity should be less affected than propagating HOMs by variing the peripheral boundary conditions within the waveguide pipe due to their vanishing field amplitudes in the end cells of the cavity. Changed peripheral boundary conditions are realized experimentally by movable shorts within beam pipes driven by stepper motors. Information about the field distribution of the trapped modes was delivered by an on axis bead pull measurement device. Therefore a nylon thread, driven by a stepper motor, was fed through the structure carrying a spherical dielectric bead in order to perturbate transversal and longitudinal electric field in like manner. The dielectric sphere measures the square of the value of the electric field strength at the position of the sphere. The nylon thread could be fed only through a small on axis hole without obstructing the movability of the shorts. The bead-pull measurements were suited to get the on axis electric field distribution. Therefore off axis measurements which are necessary to get the shuntimpedances of dipole modes were not possible but they were calculated by MAFIA for dipole modes as well as for monopole-TM modes. The trapped modes were excited by very small loops (to 
minimize their own influence on the field distribution), which dived into the cells where we expected the strongest field amplitudes of the trapped mode under investigation. All RF-measurements were performed using a HP 8720A Network Analyzer (130 MHz$20 \mathrm{GHz}$ ). Figure 1 illustrates a schematical drawing of the RF-Test-Setup with a 9-cell copper TESLA cavity with beam pipes.

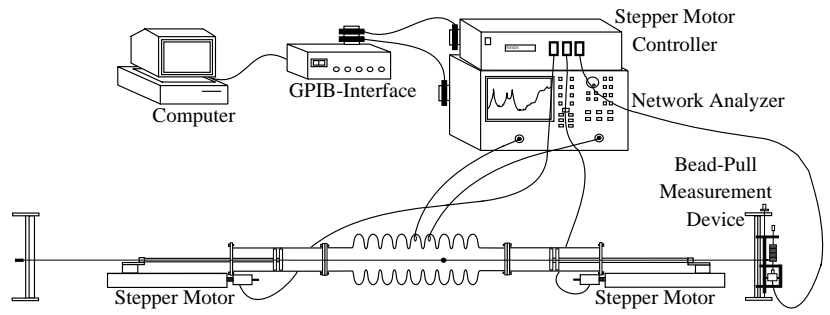

Figure 1: Drawing of the measurement setup with the TESLA 9-cell structure, the movable shorts in the beam pipes and the on axis bead-pull measurement device.

\subsection{Measurement Method}

We measured the transmission signal $\left(\mathrm{S}_{21}\right)$ between two coupling loops. A trapped mode can only be localized in frequency regions, where the transmission signal remains unaffected or shows only small changes for different positions of the movable shorts, which indicates clearly low coupling to the beam pipes and thus also low coupling to any kind of HOM dampers. At those frequency regions bead-pull measurements were performed in order to verify the existence of a localized electric field distribution. This method was proposed earlier [3] but a main problem arises since the passbands tend to overlap even for high frequencies. As a consequence we would not be able to find all possible trapped modes with this method. We therefore started to make numerical calculations as described below using the MAFIA code [4], whereby the problem of overlapping passbands vanishes. Using the numerical results we were able to verify trapped modes faster and more precisely with bead-pull measurements since we have additional information about the frequency, the field distribution and the azimuthal type of the trapped modes (i.e. monopole modes, dipole modes etc.). With automized measurements we could examine a frequency range up to $10 \mathrm{GHz}$, for which we got reliable results given by MAFIA allowing for the performance limit of our computer system.

\subsection{Numerical Calculations}

The method to find trapped modes with MAFIA is very similar to the method proposed for the measurements. We looked for the frequency differences of comparable modes found with the MAFIA eigenmode solver in two separate runs using two different boundary conditions at the end of the beam pipes. A large dependence of the calculated two frequencies of one mode indicates a strong field amplitude, whereas a small frequency shift of a given mode indicates low or even no field amplitudes within the beam pipes (comp. Fig. 2 and Fig. 4). For the first run we assumed ideal electric and in a second run ideal magnetic boundary conditions at the end of the beam pipes. We chosed a beam pipe length of $3 / 4 \lambda_{\text {fund }}$ on both sides of the cavity. This will not strongly perturbate the field of modes below cutoff with evanescent field amplitudes nearby the end cells within the beginning part of the beam pipes, like the fundamental accelerating mode at $1.3 \mathrm{GHz}$.

\section{RESULTS}

\subsection{Monopole-TM Modes}

Figure 2 shows the amount of frequency shift $\Delta \mathrm{f}$ depending on the boundary conditions versus mode number in order as they appear in the calculation. For trapped modes we have to look at local minima. The sudden step of $\Delta \mathrm{f}$ at mode no. 28 expresses the presence of the first beam pipe cutoff for monopole-TM modes $\left(\mathrm{f}_{\text {TM01 }}=2.95 \mathrm{GHz}\right.$ for a pipe radius of $\left.39 \mathrm{~mm}\right)$. We find a very small "hill" just before the sudden step in the third passband (mode no. 19-27), which is located completely below the beam pipe cutoff, demonstrating the sensibility of this method to evanescent fields.

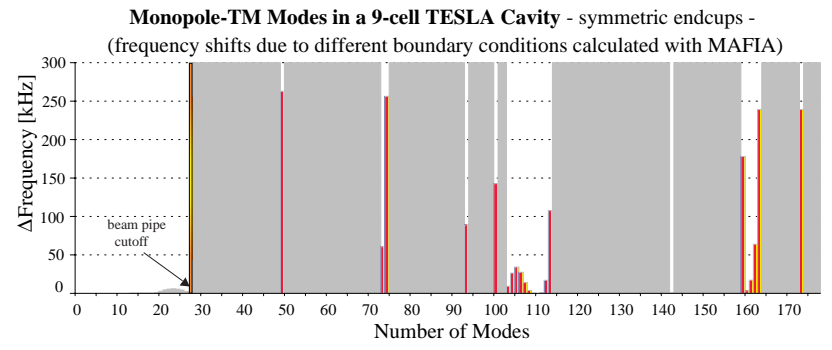

Figure 2: The Monopole-TM Modes in a 9-cell TESLA cavity calculated in two runs with different boundary conditions (see text!). Shown is the frequency shift $\Delta \mathrm{f}$ in $[\mathrm{kHz}]$ in dependence on the continuous number of calculated modes.

The first local minimum appears at mode no. 49, of which the calculated field distribution and the measured electrical field profile are presented in Fig. 3 as an example. At the top we see the electric field amplitude versus the position of the bead in arbitrary units. At the bottom the calculated field distribution is shown, which enables the reader to compare the position of the bead with the position in the cavity. The reason for the asymmetric field profile (comp. also Fig. 5) is due to small deviations from the perfect cell geometry caused for example by small fabrication errors or mechanical tensions. Relevant parameters of trapped Monopole-TM modes with the highest longitudinal shuntimpedances 
above the beam pipe cutoff are given in Table 1 (mode numbers related to Fig. 2).

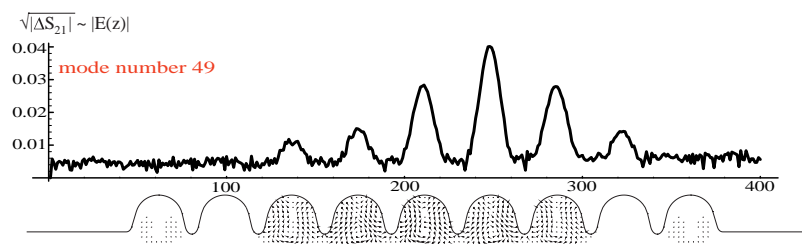

Figure 3: Electric field profile of trapped Monopole-TM mode no. 49 calculatad at $\mathrm{f}=3.855 \mathrm{GHz}$ (bottom) and measured at $\mathrm{f}=3.854 \mathrm{GHz}$ (top).

Table 1: Cavity parameters calculated by MAFIA

\begin{tabular}{|c|c|c|c|c|}
\hline $\begin{array}{c}\text { Mode } \\
\text { Number }\end{array}$ & $\begin{array}{c}\text { Frequency } \\
\mathbf{f}[\mathbf{G H z}]\end{array}$ & $\begin{array}{c}\text { Quality Factor } \\
\mathbf{Q}\end{array}$ & $\begin{array}{c}\text { Longitudinal Shunt- } \\
\text { impedance } \mathbf{R}_{\mathbf{s}} \mathbf{[}[\mathbf{k} \boldsymbol{\Omega}]\end{array}$ & $\begin{array}{c}\text { Characteristic Impedance } \\
\mathbf{R}_{\mathbf{s}}^{\boldsymbol{\|}} / \mathbf{Q}[\Omega]\end{array}$ \\
\hline 49 & 3.855 & 35704 & 1226.3 & 34.35 \\
\hline 93 & 5.461 & 62639 & 599.2 & 9.57 \\
\hline 105 & 5.902 & 36396 & 73.7 & 2.03 \\
\hline 106 & 5.912 & 37982 & 330.6 & 8.70 \\
\hline 107 & 5.922 & 40022 & 124.5 & 3.11 \\
\hline 142 & 6.687 & 44049 & 210.3 & 4.78 \\
\hline
\end{tabular}

\subsection{Dipole Modes}

Analogous simulations had been made for dipole modes. The most trapped dipole modes can be found, where low frequency shifts occur in Fig. 4. In Table 2 we summarize relevant parameters for trapped dipole modes with the highest transversal shuntimpedances above the cutoff of the beam pipe.

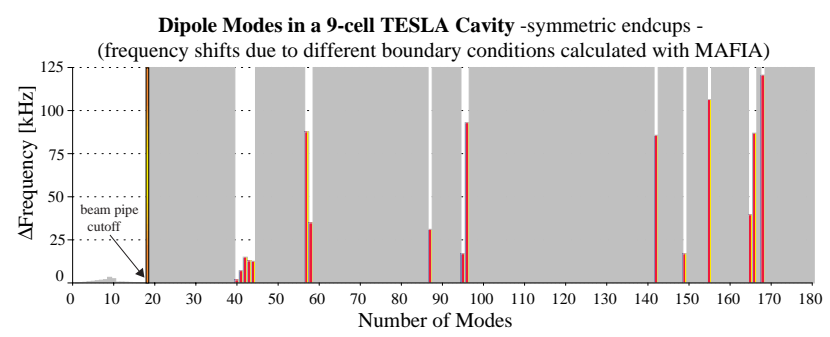

Figure 4: Results for dipole modes. Compare caption of Fig. 2 for details.

Table 2: Cavity parameters calculated by MAFIA

\begin{tabular}{|c|c|c|c|c|}
\hline $\begin{array}{c}\text { Mode } \\
\text { Number }\end{array}$ & $\begin{array}{c}\text { Frequency } \\
\mathbf{f}[\mathbf{G H z}]\end{array}$ & $\begin{array}{c}\text { Quality Factor } \\
\mathbf{Q}\end{array}$ & $\begin{array}{c}\text { Transversal Shunt- } \\
\text { impedance } \mathbf{R}^{\perp}{ }_{\mathbf{s}}[\mathbf{k} \Omega \mathbf{]}\end{array}$ & $\begin{array}{c}\text { Characteristic Impedance } \\
\mathbf{R}_{\mathbf{s}}^{\perp} \mathbf{Q}[\Omega]\end{array}$ \\
\hline 43 & 3.088 & 74926 & 54.1 & 0.72 \\
\hline 44 & 3.091 & 75539 & 196.7 & 2.60 \\
\hline 60 & 3.359 & 39427 & 2.4 & 0.06 \\
\hline 87 & 4.323 & 62268 & 6.9 & 0.11 \\
\hline 149 & 5.651 & 92669 & 5.7 & 0.06 \\
\hline 165 & 5.768 & 102813 & 3.7 & 0.04 \\
\hline
\end{tabular}

The first two passbands (mode no. 1-18) are located below the cutoff $\left(\mathrm{f}_{\mathrm{TE} 11}=2.25 \mathrm{GHz}\right)$, thus evanescent field amplitudes in the beam pipes cause only small frequency shifts. The first trapped mode above cutoff (mode no. 40) is the first mode of a whole passband showing only small amount of field strength in the beam pipes. Some examples of trapped modes are shown in Fig. 5 (mode no. 40, 87 and 95 from top to bottom).

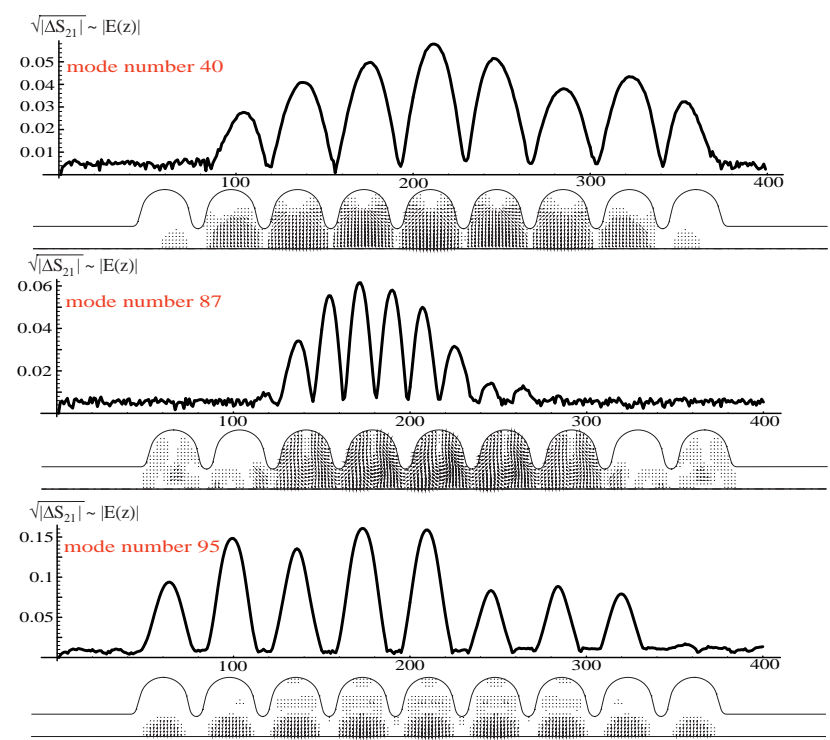

Figure 5: Trapped dipole mode (comp. Figure 4) no. 40 ( $\mathrm{f}=3.084 \mathrm{GHz}$ MAFIA; $3.078 \mathrm{GHz}$ meas.), mode no. 87 (f = 4.323 GHz MAFIA; $4.314 \mathrm{GHz}$ meas.) and mode no. 95 ( $\mathrm{f}=4.426 \mathrm{GHz}$ MAFIA; $4.421 \mathrm{GHz}$ meas.).

\section{CONCLUSION AND OUTLOOK}

We proposed a method to measure trapped modes in TESLA cavities by using the information of numerical calculations. The numerical method avoids the in reality existing problem of overlapping passbands and delivers the field distributions of trapped modes and the frequencies for more accurate RF-(bead-pull)measurements. The measurement results given are in good conformity with the numerical simulations. Field profile comparisions of several trapped modes within a frequency range of $10 \mathrm{GHz}$ had been verified and their beam dynamic related parameters like quality factors and shuntimpedances were calculated. Numerical calculations for the current TESLA cavity design with asymmetric endcups [1], which helps to reduce the unwanted trapped modes, have been carried out and have to be proved with a copper model which will be delivered from DESY in the near future.

\section{REFERENCES}

[1] TESLA and SBLC Collaboration: "Conceptual Design Report for a $500 \mathrm{GeV}$ Linear Collider with Integrated X-Ray Laser Facility", DESY Print, December 1996

[2] T. Weiland, U. van Rienen, P. Hülsmann, W.F.O. Müller, H. Klein "Investigations of higher order modes using a 36-Cell test structure", Physical Review Special Topics - Accelerator and Beams, March 1999

[3] F. Marhauser, H.-W. Glock, P. Hülsmann, M. Kurz, H. Klein: "Search for Trapped Modes in TESLA Cavities", 1997 Particle Accelerator Conference, Vancouver, B.C., Canada, May 1997

[4] T. Weiland et al.: "Solutions of Maxwells's Equations using the Finite Integration Algorithm”; Version 3.2; Darmstadt 1993 